\title{
CHANGING DRIVER BEHAVIOR THROUGH UNCONSCIOUS STEREOTYPE ACTIVATION
}

\author{
Rob Gray \& Russ Branaghan \\ Department of Applied Psychology \\ Arizona State University \\ Mesa, AZ USA \\ Email: robgray@asu.edu; Russell.Branaghan@asu.edu
}

\begin{abstract}
Summary: Under the guise of evaluating a head-up display in a driving simulator, participants completed scrambled sentence tasks (while waiting at stop signs) designed to prime either an elderly or teenage stereotype. Driving speeds between stop signs in the Stereotype conditions were compared to Control conditions in which age non-specific words were substituted for stereotyped words. Participants had a lower maximum speed in the Elderly Stereotype condition and a higher maximum speed in the Teenage condition (as compared to controls). These effects were obtained even though the participants were completely unaware of the themes in the experimental conditions. For both stereotypes, the change in behavior occurred relatively quickly: a significant effect on driving speed was observed after only five stops. These findings indicate that it may be possible to reduce the incidence of dangerous driving behavior through the use of unconscious priming.
\end{abstract}

\section{INTRODUCTION}

How can we encourage people to drive more safely? Risk homeostasis theory (Wilde, 1988) suggests that when a driver is aware of a safety intervention it may be limited in its effectiveness because behavior may be altered to maintain a constant level of risk. Can we change driver behavior without the driver's awareness? Recent research suggests that this may indeed be possible.

Non-conscious activation of a stereotype about a social group can affect both simple and complex motor behaviors, even for individuals who are not members of the stereotyped group (Bargh, et al., 1996). For example, we found that non-consciously priming an elderly stereotype led younger drivers to reduce their driving speed (Branaghan \& Gray, 2008). Under the guise of evaluating a head-up display in a driving simulator, participants completed scrambled sentence tasks (SSTs) while waiting at stop signs. In these sentences one word was related to an elderly stereotype (e.g. bingo, grey, Florida). Driving speed and driving time between stop signs in this Elderly Stereotype condition were compared to a Control condition in which age non-specific words were substituted for elderly stereotyped words. Participants had a lower maximum speed and longer driving time in the Elderly Stereotype condition than in the Control condition. This effect was obtained even though the participants were completely unaware of the theme in the experimental condition. An open-ended question in the post task questionnaire provided no evidence that participants were aware of the elderly primes. Further, a forced-choice recognition question yielded no evidence that participants were aware of the Elderly theme in the Elderly Stereotype condition. It may be possible to apply these findings to improve perceptual-motor control in dangerous situations (e.g., reducing driving speed in teenage drivers). 
In the present study we extended this previous work by: (i) investigating the time course of the stereotype activation effect, and (ii) comparing the relative effects of a teenage and elderly stereotypes on driving behavior.

\section{METHODS}

\section{Driving Simulator}

The DS-600c Advanced Research Simulator by DriveSafety ${ }^{\mathrm{TM}}$ was used. This simulator was comprised of a 300 deg wraparound display, a full-width automobile cab (a Ford Focus) and a motion platform. Tactile and proprioceptive feedback cues are provided via dynamic torque feedback from the steering wheel and vibration transducers mounted under the driver's seat. The motion platform provided coordinated inertial cues for the onset of longitudinal acceleration and deceleration. The DriveSafety ${ }^{\mathrm{TM}}$ software captured various driving performance elements at 60 $\mathrm{Hz}$.

The driving course was a two-lane rural road with several curves and hills. Ten stop signs (and accompanying stop lines) were randomly placed along the roadway. There was no other simulated traffic on the roadway during the experiment.

\section{Procedure}

After a short practice session designed to allow drivers to be comfortable with the simulator, each driver completed two course runs corresponding to the two conditions: Stereotype, and Control. For both of these conditions drivers were instructed to bring the car to a complete stop at each of the 10 stop signs along the course. When the car reached a velocity of $0 \mathrm{mph}$, three sets of five words each (presented at the top, middle and bottom of the screen in green text) were superimposed on the display of the driving scene. Drivers were instructed to rearrange the words in each set to make a four-word sentence and say this sentence aloud. Once this scrambled sentence task (SST) was completed they were instructed by the experimenter that they could proceed to the next stop sign and the word sets were removed from the screen. After the tenth and final SST was completed, drivers were instructed to drive until the simulation ended. Drivers were instructed that they could drive at any speed that felt comfortable to them and there were no speed limit signs.

In the present study, the word sets developed by (Bargh, Chen et al. 1996) were used. For the Elderly Stereotype condition, these sets each contained words that are associated with the elderly but did not reference driving or slowness. For example, "from are Florida oranges temperature", "sky the seamless gray is", and "are we stubborn courteous sometimes". For the Elderly Control condition, the word sets contained age non-specific words in place of the elderly stereotyped words. For example, "from are Ohio oranges temperature", "sky the seamless blue is", and "are we friendly courteous sometimes". For the Teenage Stereotype condition, these sets each contained words that are associated with teenagers but did not reference driving. For example, "the interesting date was door" and "sophomore attended the late it". For the Teenage Control condition, the word sets contained age non-specific words in place of the teenage stereotyped words. For example, "the interesting book was door" and "worker attended the late it". 
Half of the drivers completed the Elderly condition and half of the drivers completed the Teenage condition. In both cases, drivers completed both the stereotype and control conditions with the order counterbalanced across participants. The order of presentation of word sets for each run was random.

\section{Post-test Questionnaire}

Following each driving episode, participants were administered a questionnaire inquiring what they noticed about the experiment and the materials. The first question asked if they had noticed any words or themes that many of the sentences had in common and if so, what were those themes? The second question informed the user that many of the sentences had words associated with a common theme, and asked them to choose which theme they thought it was from a list of six items (i) Food, (ii) Weather, (iii) Travel, (iv) Sports, (v) Youth, or (vi) Old Age.

\section{Participants}

Twenty-four drivers (ages 25-41) with 7-21 years of driving experience participated in the experiment. Twelve of the drivers completed the Elderly condition while 12 completed the Teenage condition.

\section{Data Analysis}

The dependent variable used in this experiment was the maximum driving speed between each of the stop signs.

\section{RESULTS}

Figure 1 plots the mean maximum speed (averaged across the 12 drivers in each condition). Consistent with our previous findings (Branaghan \& Gray, 2008), maximum speed was lower in the Elderly stereotype condition than for the associated control condition. It can also be seen in this figure that the opposite effect occurred for the Teenage stereotype: maximum speed was higher in the Teenage stereotype condition as compared to its associated control. Pairwise t-tests revealed that the maximum speed was significantly lower in the Elderly condition than in the associated control condition $[\mathrm{t}(11)=4.2, \mathrm{p}<0.01]$ and the maximum speed was significantly higher in the Teenage condition than in the associated control condition $[\mathrm{t}(11)=-5.1, \mathrm{p}<0.01]$. 


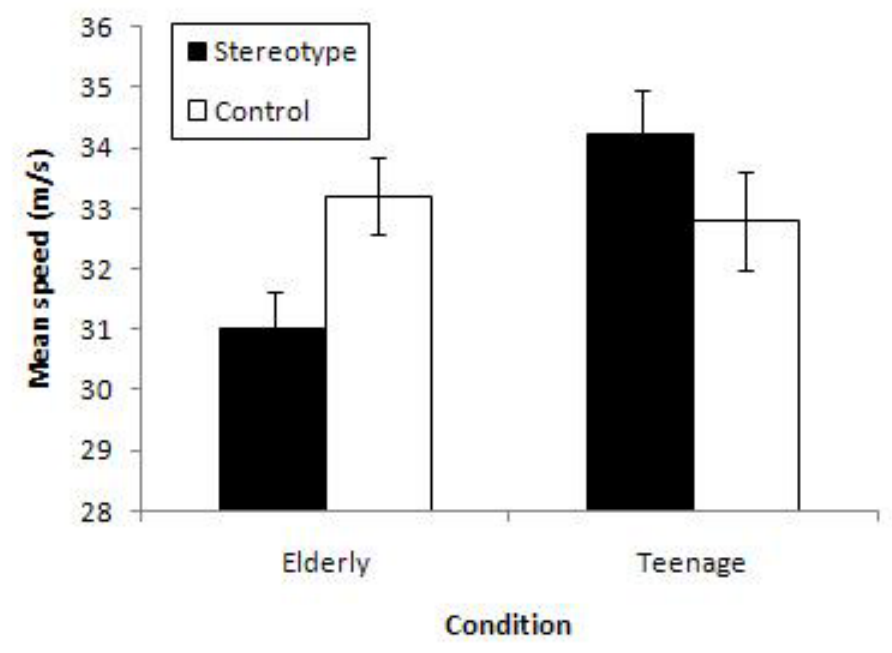

Figure 1. Mean maximum driving speed averaged across the 12 drivers in each condition. Error bars are standard errors.

How quickly did these changes in driver behavior occur? To address this question we calculated the difference in maximum driving speed for the stereotype and control conditions for each of the 10 stop signs separately. Figure 2 plots the mean speed differences (averaged across the 12 drivers in each condition). A $2 \times 10$ mixed factor ANOVA with stereotype (Elderly vs Teenage) as the between subjects factor and stop\# (1-10) as the within-subject factor revealed a significant main effect of stereotype $[F(1,11)=8.7, p<0.05]$ and a significant stereotype $x$ stop \# interaction $[\mathrm{F}(9,198)=14.2, \mathrm{p}<0.001]$. Post-hoc comparisons revealed that the speed difference was significantly different than 0.0 for stops $6-10$ in both the elderly and teenage stereotype condition (p all $<0.05$ ).

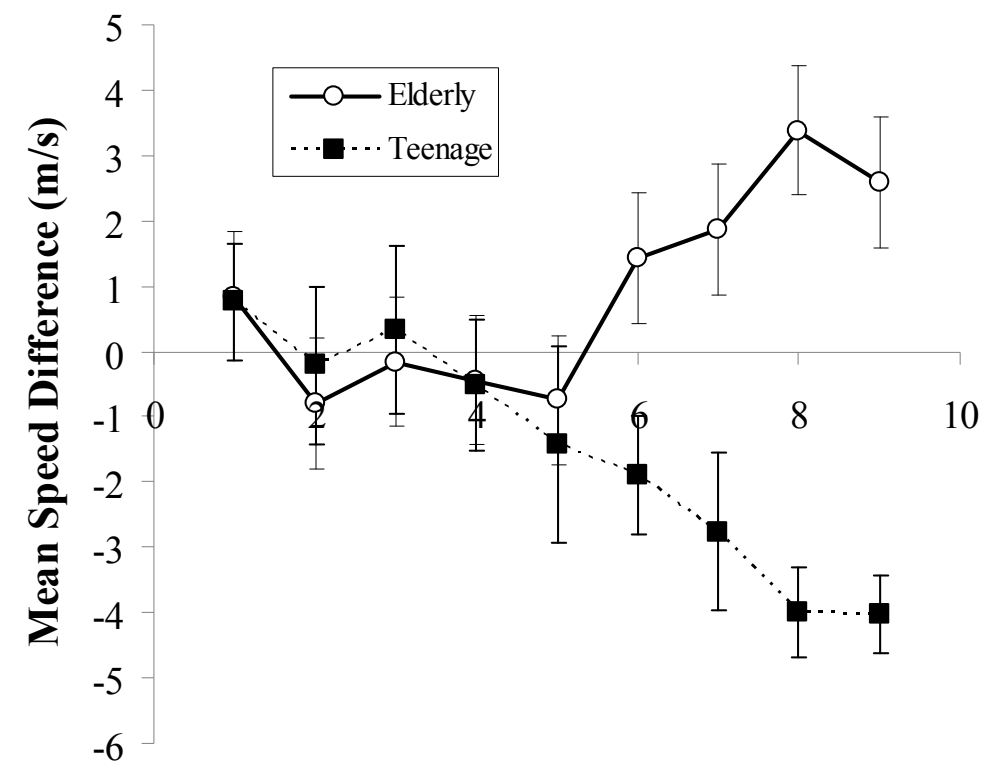

Stop \#

Figure 2. Mean difference between maximum speeds in the stereotype and control conditions. Error bars are standard errors. 
The open-ended question asking if the participants detected any words or themes that the sentences had in common yielded only a few responses. Twenty of the 24 total participants answered no, they did not detect any themes. None of the four participants that identified a theme did so correctly.

Figure 3 shows the frequency of responses to the question "Many of the sentences had words associated with a common theme. Of the list below, which theme do you think that was?" As predicted, only one participant in the Elderly stereotype group chose old age as the theme reflected in the sentences and only one participant in the Teenage stereotype group chose youth as the theme reflected in the sentences.

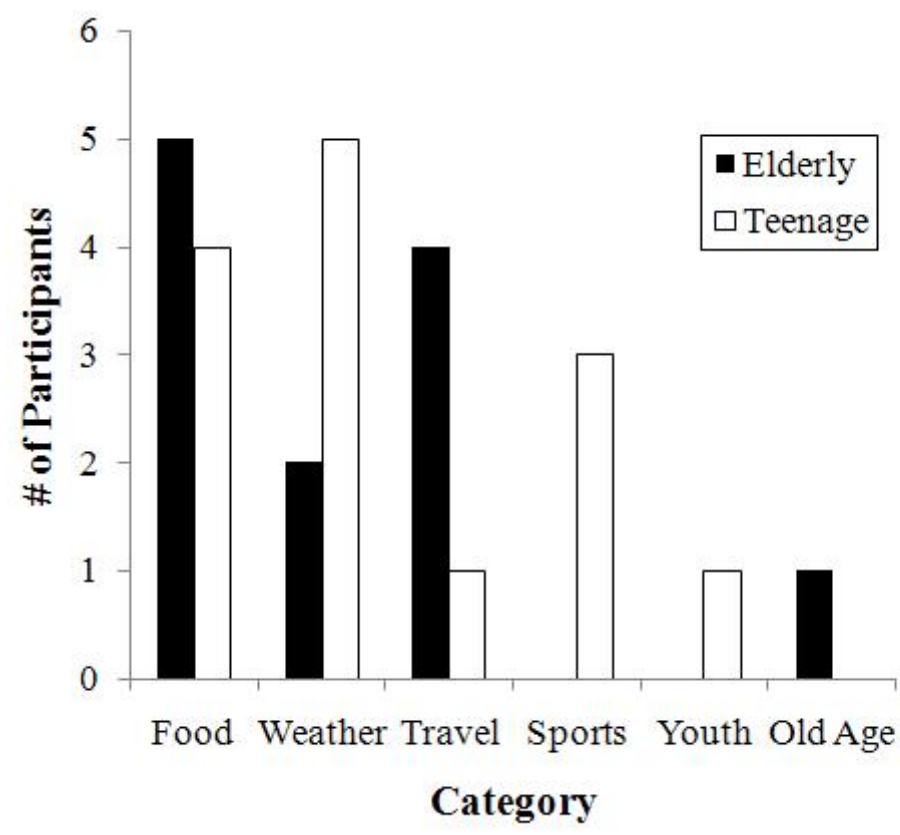

Figure 3. Theme choice in post-driving questionnaire.

\section{CONCLUSIONS}

Priming a stereotype via the SST affected driving performance in manner consistent with our previous research (Branaghan \& Gray, 2008). Participants drove slower between stop signs when primed with elderly stereotypes and faster between stop signs when primed with teenage stereotypes. Importantly, these effects were obtained even though the participants had no conscious awareness that they were being primed by a stereotype. An open-ended question in the post task questionnaire provided no evidence that participants were aware of the either the elderly or teenage primes. Further, a forced-choice recognition question yielded no evidence that participants were aware of either the Elderly or Teenage theme in the stereotype conditions.

Changes in driving behavior resulting from stereotype priming occurred relatively quickly. For both the Elderly and Teenage stereotypes a significant difference in driving behavior was observed after only five stops. This is equivalent to 15 priming words and roughly 5 minutes of driving. 
These findings raise the possibility of several exciting avenues for application in the area of driving safety. Would it be possible to develop a practical, real-world analog of the scrambled sentence stimuli that could prime stereotypes in real driving? Can these technique be extended to other aspects of driving behavior besides speed choice (e.g., seatbelt use, using turning signals, etc)? Can we use non-conscious means to improve vigilance or reduce fatigue?

\section{ACKNOWLEDGEMENTS}

This work was supported by the National Science Foundation Faculty Early Career Development Program (Award \# 0239657 to author R.G.).

\section{REFERENCES}

Bargh, J. A., M. Chen, et al. (1996). Automaticity of social behavior: Direct effects of trait construct and stereotype activation on action. Journal of Personality and Social Psychology, $71,230-244$.

Branaghan, R. \& Gray, R. (2008). Non-conscious activation of an elderly stereotype leads to safer driving behavior. Proceedings of the Human Factors and Ergonomics Society $52^{\text {nd }}$ Annual Meeting.

Wilde, G. J. S. (1988). Risk homeostasis theory and traffic accidents - propositions, deductions and discussion of dissension in recent reactions. Ergonomics, 31, 441-468. 